\title{
Exploration of model coupling strategies in a hybrid agent-based traffic simulation
}

\author{
Jean-François Erdelyi ${ }^{10000-0002-4384-5922]}$, Frédéric Amblard ${ }^{10000-0002-2653-0857]}$, Benoit \\ Gaudou $^{1[0000-0002-9005-3004]}$, Elsy Kaddoum ${ }^{2[0000-0003-4610-4174]}$, and Nicolas \\ Verstaevel $^{1[0000-0002-7879-6681]}$ \\ 1 UMR 5505 IRIT, Université Toulouse 1 Capitole, France \\ 2 UMR 5505 IRIT, University of Toulouse, France \\ Contact Author: jean-francois.erdelyi@irit.fr
}

\begin{abstract}
Traffic simulation is a tool used by urban planners to assess the impact of new urban designs and public policies on mobility. Over the years, numerous traffic models have been proposed, each model offering different levels of details and performances. Multi-level model coupling is an interesting approach to combine the advantages of complementary representations while limiting their drawbacks. In this paper, we design and evaluate the performances of hybrid traffic models combining a microscopic model (IDM) with a mesoscopic model (event-driven and queue-based). The results show that microscopic models have more diversity in terms of behaviors but reduce the vehicle average speed and mesoscopic models are more efficient in terms of computational time but display a higher vehicle speed. Their hybridization then enables to find a balance between scalability and the variety of the observed behaviors.
\end{abstract}

Keywords - Traffic simulation, model composition, GAMA platform.

\section{Introduction}

The sustainable management of mobility is identified as a key component for a wellfunctioning Smart City [25]. On one side, technologies are evolving offering new and more effective transportation modes [22]. On the other side, urban planners see mobility as an enabler for designing better cities, integrating social, environmental, and economical components [7]. Therefore, there is an increasing need for tools that allow to assess the impact of these disruptive innovations based on "what if" prospective studies [31].

Various traffic simulation models and software tools have been developed to specifically model, plan and analyze current traffic in terms of offer and demand [20]. Each tool is focusing on specific aspects of traffic modeling using dedicated modeling approaches. A microscopic simulation focuses on modeling each vehicle and its dynamics individually, whereas a macroscopic simulation focuses on aggregated information such as traffic density and traffic flow [16]. To study the impact of urban planning decisions, that requires to both observe large-scale traffic at the scale of a metropolis and focus on the microscopic traffic at the scale of a ward, it is necessary to design tools that can combine and switch between those different scales at runtime [13]. Furthermore, it is also interesting to combine those different models with other models to study for example the impact on environmental factors [24], or the individual response of citizens to catastrophic events [8].

Large-scale road traffic in a city, including individual choices in terms of mobility, is a complex system: it is composed of numerous heterogeneous entities with nonlinear interactions that are geographically distributed and can be modeled and/or observed at different 
levels [13]. The agent-based paradigm is thus a particularly suitable approach to model such complex phenomena [4] and to couple various models, being of the same phenomenon at different scales or different phenomena [11]. The key issues when coupling different models (in particular of the same phenomenon at different levels) are to control the side effects at the interaction between models and to avoid divergence of the results [2].

This paper studies the impact of coupling different models of traffic simulation within an agent-based framework. One microscopic (IDM [10]) model and one mesoscopic (eventdriven and queue-based [9]) model have been implemented and coupled on a road network: moves of vehicles on each road segment can follow the former or the latter model. The main contribution of this paper is to evaluate the impact of the hybridization (i.e. the ratio of each model type) on simulation performances (computation time, vehicles' average speed, and vehicles' time to reach their target). In short term, the objective is to understand and quantify the pros and cons of this hybridization and to emphasize the need for an equilibrium between computation time of the simulation and variety of agents' behaviors, and identify challenges for dynamic model coupling. In long term, the objective is to propose a framework to offer the possibility of dynamic model coupling applied to mobility simulation.

The paper is organized as follows: Section 2 provides background on traffic simulation and models coupling, Section 3 gives details about the two traffic flow models and their coupling, Section 4 analyses the performance of the integrated coupling of these two models. Finally, Section 5 concludes with perspectives.

\section{Related work}

Numerous road traffic models have been proposed. Each model is designed with a specific hypothesis, depending on the simulation objectives and computational limits. Those models can be classified into three modeling levels [14]:

1. Microscopic [12,29]: such models describe individually the entities involved in the traffic (vehicles, pedestrians, etc.), their interactions, and lead to emerging phenomena (such as congestion). They are considered the most accurate and realistic. However, this representation has some limitations especially when upscaling: the computation cost indeed evolves rapidly with the number of individuals $[27,28]$. These models are also difficult to calibrate because of their large number of parameters.

2. Macroscopic [18]: All individual vehicles are here aggregated and such models focus more on macroscopic variables of average speed, flow, and vehicle density [15]. The observed behaviors are thus less precise. However, they are very effective for representing large-scale phenomena in space and time. These models often use probabilistic concepts, which makes them simple to describe and calibrate. About traffic, they use concepts from fluid mechanics, including the conservation laws [21] and differential equations.

3. Mesoscopic [6]: these models consider an intermediate representation between microscopic and macroscopic models [30]. Two main approaches can be found in the literature: either the individuals are grouped and thus individual behaviors are not taken into account, or the dynamics of the flow of entities is determined by a simplification of the individual dynamics and managed at the road level $[3,28]$. The advantage of this approach is to have an intermediate between the microscopic fine representation of individual vehicles, but expensive and difficult to calibrate, and macroscopic inexpensive and simple simulations providing coarse results. 
Each of these modeling approaches has thus its specificities: macroscopic models are particularly relevant for large-scale simulations (e.g. the scale of a metropolis) whereas microscopic ones can be applied to represent precise traffic in a small ward. When a model is designed for urban planning purposes, it would be interesting to be able to observe both levels. To this extends it appears necessary to combine different models.

Multi-level model coupling is a technique used to combine the advantages of complementary representations of the same system. There are three main types of model coupling $[19,11]$ : integrated (model as a new model from the combination of two, or more, models), weak (the model as a set of interconnected independent models), and strong (model as a set of parallel models sharing data during the simulation). Model coupling, even if it can be technically complex when the number of interactions increases, provides huge benefits in terms of modularity: coupled model could be switched to increase precision or conversely to reduce computational time. The coupling can also be static or dynamic [28]. When the coupling is static, the coupled models and the ways they interact are defined at the initialization of the simulation and cannot be changed at runtime. This is the most commonly used approach as it does not need to implement transfer functions at runtime. The dynamic coupling approach allows the system to switch between multiple representations dynamically depending on specific criteria, such as the CPU load or user needs.

In this paper, we focus on a static hybrid traffic models, i.e. a model coupling different (mesoscopic and microscopic) representations of the same phenomenon: we divide the roads into sections and a specific (microscopic or mesoscopic) model is associated to each of them, the transition is managed by the frontier between each section.

Several works have already investigated the possibility to design a hybrid model of traffic flow, coupling models at various scales (in general a microscopic model with a mesoscopic or macroscopic model). Such models are limited to 2 or 3 road sections, each section using either a microscopic or a mesoscopic model. The objective is to ensure the coherence between these various models: in particular that the number of vehicles or the flow speed is not altered at the transition between models [2]. [17] focuses on the compatibility between a micro and a macro model and in particular on the compatibility of a micro car-following model at the interface with a macroscopic model. [5] also investigates how to minimize the perturbations at the interface between 2 levels. [1] focuses more specifically on the switch from a macroscopic to a microscopic model by proposing probabilistic desegregation, which could open the door to dynamic hybrid models.

Whereas previous models only focus on the interfaces between a microscopic and a macroscopic model, we go one step further by investigating and quantifying the impact of the hybridization rate, i.e. the rate of mesoscopic road segments in a hybrid model.

\section{Methodology}

This section details the design of an agent-based traffic model of a highway road. The road is sliced into segments of $1 \mathrm{~km}$, where each segment is using its own traffic model. The objective of the simulations is to study the impact of hybridizing different types of traffic models within the same simulation by analyzing vehicles' average speed, vehicles' time to reach their target, and computation time for each simulation. The rest of this section is organised as follows: subsection 3.1 introduces the two models used for modelling traffic: one microscopic model (IDM model [10]) and one mesoscopic model (event-driven and queuebased [9]), and subsection 3.2 describes the implementation of those models within the agent-based modeling and simulation GAMA platform [26] and the coupling mechanism. 


\subsection{Traffic models}

Micro model: Intelligent Driver Model (IDM) [29]. The first model is a microsimulation model that simulates the individual behavior of a vehicle on a road. IDM is a time-continuous car-following model that describes the evolution of the position and velocity of each individual vehicle: it models a vehicle acceleration and deceleration, following the principle that a vehicle tries to maintain a minimum gap with its front vehicle. The original version of IDM is used in this experimentation. However, a variation of the model can be found that includes other factors such as the human reaction time [10]. This model is described by [10] as follows.

The computation of the $n^{\text {th }}$ vehicle acceleration $\dot{v}_{n}$ is given by the equation:

$$
\dot{v}_{n}=a_{n}\left(1-\left(\frac{v_{n}}{v_{n}^{0}}\right)^{4}-\left(\frac{s^{*}\left(v_{n}, \Delta v_{n}\right.}{s_{n}}\right)^{2}\right)
$$

where $a_{n}$ is the maximum acceleration of the vehicle $n, v_{n}$ is the $n^{\text {th }}$ vehicle velocity, $v_{n}^{0}$ is the desired speed of the vehicle $n . s_{n}$ is the distance gap between two vehicles and is defined as: $s_{n}=\Delta x_{n}-l_{n+1}$, where $s_{n}$ is the distance bumper to bumper, $\Delta x_{n}=x_{n+1}-x_{n}$ is the distance between the vehicles $n$ and $n+1$, and $l_{n}$ the length of the vehicle $n$.

The desired minimum gap of the vehicle $n, s_{n}^{*}$, is given by:

$$
s^{*}\left(v_{n}, \Delta v_{n}\right)=s_{n}^{0}+T_{n} v_{n}-\frac{v_{n} \Delta v_{n}}{2 \sqrt{a_{n} b_{n}}}
$$

where $s_{n}^{0}$ is the jam distance of the vehicle $n$ which is the distance between vehicles in a traffic jam, $T_{n}$ is the safety time gap of the vehicle $n, \Delta v_{n}=v_{n+1}-v_{n}$ the velocity difference of the vehicle $n$, and $b_{n}$ the desired deceleration of the vehicle $n$.

The IDM model is characterized by the intuitive parameters displayed in the Table 3.1 (with the commonly used default values of parameters). It suffers some limitations such as non-realistic deceleration in case of emergency braking, and the safety gap is not enough to guaranty safety in critical situations.

\begin{tabular}{|c|c|c|}
\hline IDM parameter & IDM parameter description & Default value \\
\hline$l_{n}$ & the length of the vehicle $n(m)$ & $5 \mathrm{~m}$ \\
\hline$v_{n}^{0}$ & the desired speed of the vehicle $n(\mathrm{~m} / \mathrm{s})$ & $30 \mathrm{~m} / \mathrm{s}$ \\
\hline$s_{n}^{0}$ & the jam distance of the vehicle $n(\mathrm{~m})$ & $1 \mathrm{~m}$ \\
\hline$T_{n}$ & the reaction time of the vehicle $n(\mathrm{~s})$ & $1.5 \mathrm{~s}$ \\
\hline$a_{n}$ & the maximum acceleration of the vehicle $n\left(\mathrm{~m} / \mathrm{s}^{2}\right)$ & $4 \mathrm{~m} / \mathrm{s}^{2}$ \\
\hline$b_{n}$ & the desired deceleration of the vehicle $n\left(\mathrm{~m} / \mathrm{s}^{2}\right)$ & $3 \mathrm{~m} / \mathrm{s}^{2}$ \\
\hline
\end{tabular}

Table 1. Common default values of parameters for the IDM model

Meso model: A FIFO queue combined with the BPR flow equation. The second model is an event-driven queue-based traffic flow model [9]. Vehicles are aggregated at the road level where each road has a maximum capacity and a maximum free-flow speed so, when a vehicle enters a road, its traveling time is computed according to the current road capacity and free-flow speed using the flow equation provided by [23].

To exit a road, a vehicle has to reach 4 conditions: 
1. The vehicle is at the top of the FIFO queue.

2. The time spent by the vehicle in the queue is at least equal to the time computed by the flow equation when it entered the queue.

3. The outflow capacity of the road (the maximum number of vehicles that can travel through a road in an hour) is not exceeded.

4. The next road capacity is not exceeded.

The next road should be (by default) another meso road, in this experimentation, the next road could be also micro, Section 3.2 provides details about the micro-meso coupling.

The Bureau of Public Road (BPR) function is used to compute the flow equation [23]:

$$
t=t_{f} *\left(1+\alpha *\left(\frac{v}{c}\right)^{\beta}\right)
$$

where $t$ is the traveling time, $t_{f}=R_{n}^{d} / v_{n}^{0}$ is the free flow traveling time, $R_{n}^{d}$ the length of the road $n, v_{n}^{0}$ is the free-flow speed, $v$ the road volume, $c$ the road capacity. The $\alpha$ and $\beta$ parameters are two parameters that have to be calibrated: common values of those parameters are $\alpha=0.15$ and $\beta=4.0$. Different values of $\alpha$ and $\beta$ can be used to take into account variation in transport infrastructures such as intersection delay, stop or crosswalk.

The outflow capacity of a road is the maximum number of vehicles that can leave the road over one hour. To comply with the outflow capacity, a minimum time between two vehicles $t_{0}$ is computed using the following formula: $t_{0}=3600 / \zeta$, where $t_{0}$ is the minimum time between two vehicles and $\zeta$ is the maximum number of vehicles that can leave a road in an hour. $\zeta$ value depends on the characteristics of the road. In this paper, $\zeta$ is fixed at $2000 \mathrm{veh} / \mathrm{h}$ which corresponds to the maximum number of vehicles that can exit a segment of a one lane highway. Therefore, whenever a vehicle exit a road, the next vehicle in line in the queue will have at least to wait for a minimum time of $t_{0}$ before exiting the road itself. This model has proven to be efficient in large-scale scenarios [9].

\subsection{Implementation and coupling strategy}

The two previous models have been implemented within the GAMA platform [26].A third model (that is the object of this paper) has been implemented to couple the microscopic and mesoscopic models on a road, each road segment been controlled by one of the two traffic models ${ }^{3}$. The model is composed of two types of agents, roads and vehicles. Each vehicle moves from the origin of the road to its target, its moves are controlled by either the microscopic or the mesoscopic model depending on the road agent. Each vehicle agent is characterized by a location, speed, and acceleration and the set of parameters used in the IDM model. Each road agent is characterized by its length, maximum speed, and all the parameters related to the mesoscopic model.

An important point of the model is that, once it has been created, a vehicle agent remains in the simulation until it reaches its target: in particular, it is kept at the transition between the micro and the meso roads. The only difference between a vehicle in a meso or a micro road is the activation or not of the IDM behavior: onb a micro road, the vehicle computes its own moves following the IDM model, otherwise, the vehicle is controlled by the road. At the entrance to a meso road, the vehicle registers to the road and waits until the road agent wakes it up when it can exit the current road and enter the next road.

\footnotetext{
${ }^{3}$ In the following, we will use "meso road" (resp. "micro roa") to refer a road controlled by a mesoscopic (resp. microscopic) model.
} 
Different combinations of traffic models can be tested by selecting the model executed by each road agent, from a fully microscopic model to a fully mesoscopic model. Therefore, four types of transitions between models have to be designed to effectively couple the different models and handle the transition from one model to the other:

- From a mesoscopic model to a mesoscopic model. In this context, no specific action is required. The vehicle exiting the road just registers to the new one.

- From a microscopic model to a microscopic model. To avoid side-effects at the interface between 2 micro roads, vehicles driven by the IDM model consider a set of contiguous micro roads as a single road, and the computation of the closest vehicle, etc. will take into account vehicles of the current road, but also of the next road.

- From a mesoscopic model to a microscopic model. The key issue here is to compute the vehicle speed at the entrance in a micro road.

1. Step 1: as the vehicle is on a meso road, it does not have any speed in the sense of the IDM model. So a mean speed $v_{n}$ is computed at first: $v_{n}=R_{m}^{d} / t_{m}^{m e s o}$, where $t_{m}^{m e s o}$ is the time to travel the current meso road (considering the time in the jam, this is the true time spend on the road) and $R_{m}^{d}$ its length.

2. Step 2: it consists in detecting whether the vehicle is the leader of the platoon:

- If there is no vehicle to follow, the vehicle is the leader and it enters the road with the mean speed computed above;

- Otherwise the vehicle enters the next road with a speed that is the minimum between the mean speed (computed above) and the speed of the followed vehicle.

3. Step 3: the IDM behavior is activated, so all the interactions and behaviors are computed by the vehicle itself and the road agent is passive.

- From a microscopic model to a mesoscopic model. A vehicle from a micro road has to take into consideration some meso road capability as the inflow.

1. Step 1: if the vehicle is the leader of the platoon and the first vehicle in the road, it computes the time to reach the meso road $t_{r}=\frac{\delta d}{v_{n}}$, where $\delta d$ is the distance between the current vehicle and the end of the current road, and $v_{n}$ the vehicle speed

2. Step 2: the vehicle checks if the time to reach the next road $\left(t_{r}\right)$ is compatible with the outflow duration of the road, which means, the time to reach the next road is higher than the outflow time from the previous vehicle.

3. Step 3: if the outflow duration is compatible, the vehicle continues until the end of the current road, otherwise the vehicle is braking and comes back to Step 1.

4. Step 4: the IDM behavior is deactivated, the road takes the control of the vehicle.

\section{Experiment and Results}

The objective is to study the impact of hybridizing a microscopic and a mesoscopic simulation model on the performances of an agent-based traffic simulation. More precisely, we are interested in studying the heterogeneity of vehicle behaviors by monitoring average speed and time to travel in different traffic conditions (inflow value and rate of mesoscopic models), and the computational time required to execute the simulations.

\subsection{Experiment design}

The simulation is initialized with a $10 \mathrm{~km}$ section of motorway (segmented by $1 \mathrm{~km}$ sections) with two optional exits and one main exit (Figure 1). Each road agent is characterized by 
a length $(1 \mathrm{~km})$, a maximum speed $(34 \mathrm{~m} / \mathrm{s})$, and a model that has to be chosen between the microscopic and the mesoscopic models. Each vehicle has its set of IDM parameters initialized with values from Table 3.1.

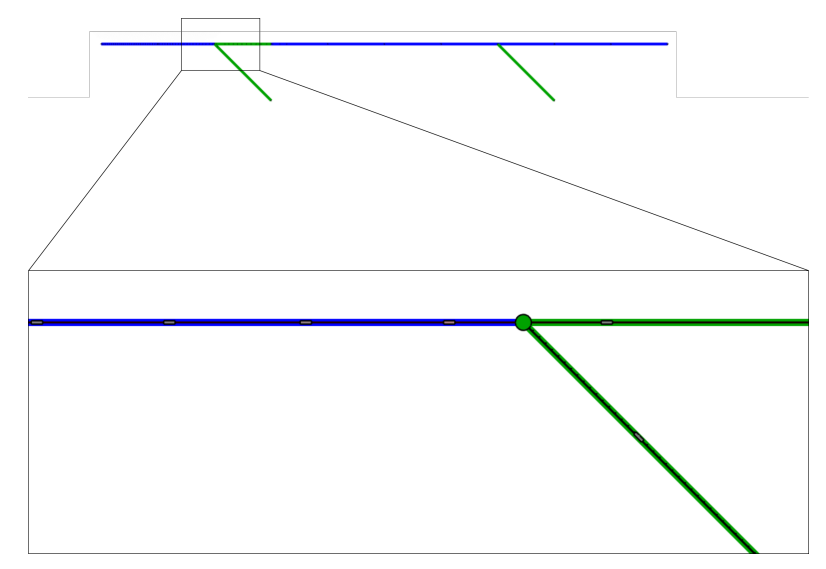

Fig. 1. Example of road configuration: blue roads are controlled by a microscopic model and green ones by a mesoscopic model. The main entrance (resp. exit) is located on the left (resp. right).

The experiment configuration is as follows:

- A simulation step lasts 0.1 seconds;

- A simulation stops after 10 minutes (of simulation time);

- 80 percent of the vehicles are going from the main entrance to the main exit and 20 percent choose randomly an alternative exit among the two other ones.

We propose to explore the model according to two parameters:

- The inflow of vehicles:it allows to adjust the number of vehicles created each step: with an inflow of 1500 , a new vehicle agent is created every 0.4 second. 5 values are evaluated: $[1500,1800,2000,2250,2400]$ where 2000 corresponds to the max outflow. The objective is to study different traffic conditions including a low traffic inflow (1500 and 1800), the maximum free-flow inflow (2000), and congested flows (2250 and 2400).

- The percentage of road hybridization, that is the rate of meso roads (micro roads are considered as the reference). 11 values are explored from $0 \%$ to $100 \%$ with an increment of $10 \%$. For each value, the roads executing the meso-model are randomly chosen.

To limit the effect of stochasticity, 20 replications (i.e. 20 simulations with the same parameters values) are executed. An exhaustive exploration is performed: all the combinations of the 11 values of hybridization and the 5 values of inflow are explored. A total of 1100 simulations (11 hybridisation values x 5 inflows x 20 runs) has thus been computed.

For each simulation, three indicators have been computed:

- Mean travel time of vehicles (in $s$ ) that exited the road during the simulation;

- Mean speed of all vehicles (in $\mathrm{km} / \mathrm{h}$ );

- Computation time (in $s$ ) required to compute the 10 minute simulations.

All simulations have been launched using a MacBook Pro with $2.3 \mathrm{Ghz}$ Intel Core i7 quad cores, 32 GB (3773Mhz) of RAM, and Intel Iris plus graphics (1536 MB). 


\subsection{Results}

As the complexity of the microscopic model is influenced by the number of vehicles traveling in the road, we first study the effect of inflow on vehicle density (with $100 \%$ of micro roads). Figure 2 plots the mean density of vehicles in the road (the difference between the number of vehicles that entered the road and the number of vehicles that exited it during the simulations) for each inflow value. The horizontal and vertical axis are respectively the time and the density, and the color represents the inflow values. The results confirm that the higher the inflow is, the higher the density is. However, we see that densities for inflows of 2400 and 2250 are similar and close to the density of value 2000. The general tendency is linear from time 0 to time 330 and the curve is starting to flatten from that time. It corresponds to the moment when the first vehicles exit the road. The lower the inflow is, the stronger is this effect. It is the consequence of road congestion: with low inflow, vehicles are driving at a higher speed. Therefore, more vehicles manage to exit the road. Whereas at higher inflow values, vehicles need more time for exiting the road, leading to congestion.

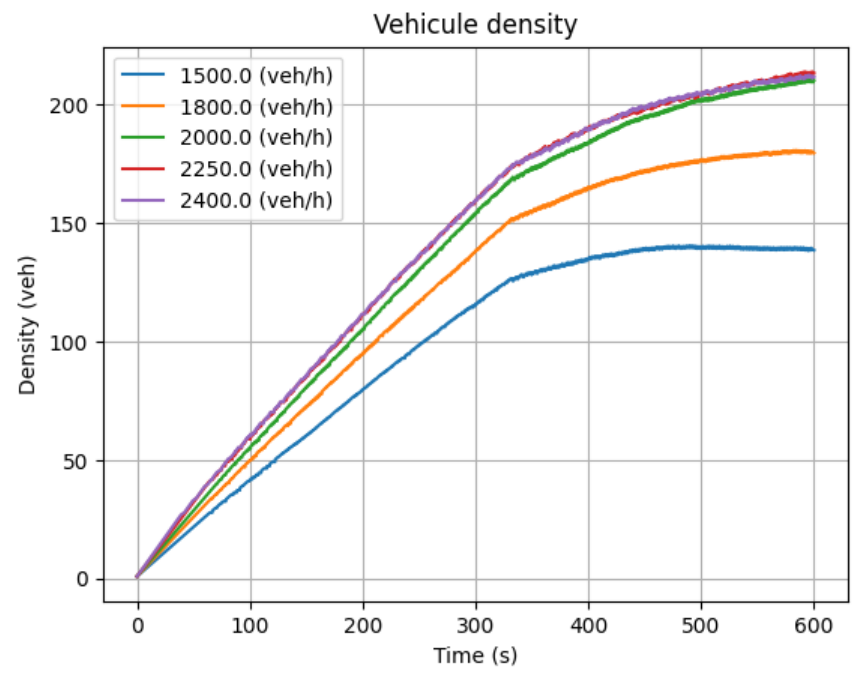

Fig. 2. Mean density of vehicles for each inflow value over time with $100 \%$ of micro roads.

On the left side, the Figure 3 plots 3D graphs displaying the distribution of the mean speed of vehicles during the travel, the time needed to compute the simulation, and the time for vehicles to travel from origin to destination (only vehicles to the main exit). The $\mathrm{x}$ and $\mathrm{y}$ axes are the parameters i.e. percentage of road hybridization and inflow values, and the z-axis displays the computed metric i.e. speed, time to travel, and computation time. The bold line is the median value, and the top and bottom lines are respectively the limits of the first and third quartiles. To ease comparisons, the right side of Figure 3 plots the evolution of the median value of each metric for each inflow value. The horizontal and vertical axis are respectively the hybridization percentage and the metric value, and the colors represent the different values of the inflow. 

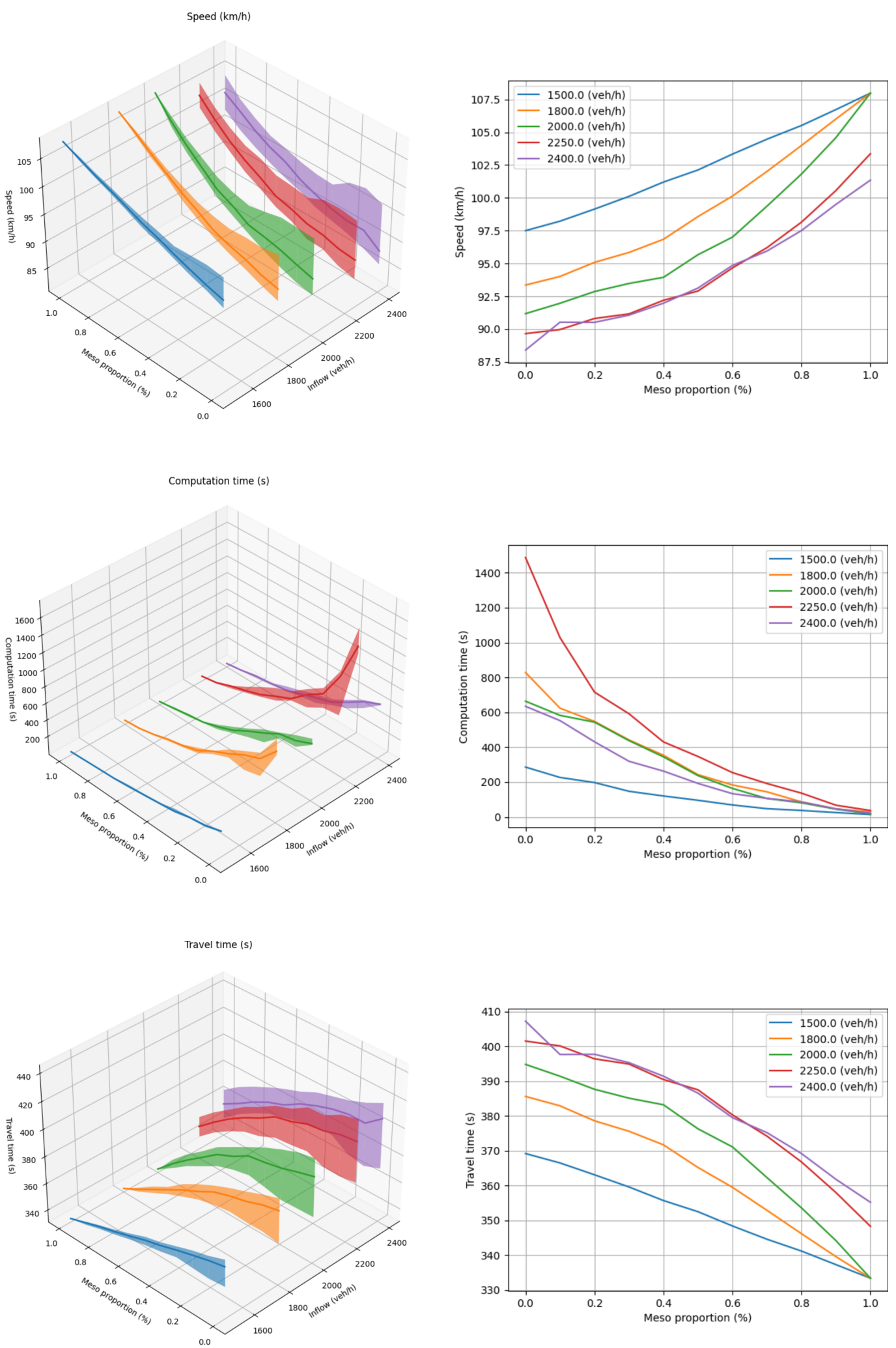

Fig. 3. On the left: the distribution of speed, computation time and time to travel for each inflow value. For each curve, the bold line corresponds to the median value, the top line the $3^{\text {th }}$ quartile and the bottom line to the $1^{\text {st }}$ quartile. On the right: only the median of the left values indicators. 
First, we can see that for any hybridization rate, an increase of the inflow leads to a reduction of the maximum speed. However, the full mesoscopic model (hybrid parameter at 1.0) produces higher speeds than the full microscopic model (hybrid parameter at 0.0). The range between Q1 and Q3 is narrower with the mesoscopic model. This could be explained by the fact that using a mesoscopic model, the vehicles tend to adopt the same behavior. On contrary, the distribution of speeds is more widespread with the micro model. As vehicles are influenced by proximity with others, and speed is influenced by acceleration and deceleration, each vehicle behaves differently. Nevertheless, we can notice that, for low inflows (below 2000), the distribution remains very limited, even for a mix of meso and micro roads (e.g. for the hybridization rate of 0.6 ).

We observe similar results with the travel times. The travel time is lower with the mesoscopic model than with the microscopic model. The distribution of the travel time is narrower with the mesoscopic model than with the microscopic model.

The results in terms of computation time confirm that for all hybridization values and all inflow values, the mesoscopic model is faster than the microscopic one. The computation time for each inflow value is similarly low when all the roads are using a mesoscopic model. However, inflow values have a strong influence on the microscopic model. This could be explained by the fact that the complexity of the microscopic model is influenced by the number of vehicles, as vehicles have to compute the distance to the closest vehicle. The more micro roads there are, the longer the computation is. Therefore, the more hybridization there is, the faster the simulation is.

From those results, we can conclude that the model coupling strategy offers an interesting approach for large-scale interactive scenarios where computation time is crucial. Each model offers different performances. Microscopic models allow to get more variations in terms of speed and time to travel, whereas microscopic models offer more gain in terms of computation time. By hybridizing the simulation and coupling both models, we can either decrease the computation time or increase the variety of observed behavior.

If we look at mesoscopic models, we observe that the inflow value has a low impact on computation time, but if we look at speed level, inflows of different values might lead to similar behaviors, and they do not produce an effect on the time to travel. On the opposite, microscopic models computation time is strongly influenced by the inflow value, but the model manages to produce different speeds for each inflow value, and therefore, produce more rich behaviors. By hybridizing those two models, it is possible to adjust those two criteria without completely deteriorating the simulation. For example, at low inflow (1500), the two models produce a relatively similar behaviors in terms of speed and travel time, but the mesoscopic model offers a better performance in terms of computational time.

While the experimentation is limited to a simple highway and needs to be experimented in real world scenarios, we see in those results the first step towards dynamic coupling of traffic models in large scale scenarios.

\section{Conclusion}

There is a growing need for simulation tools that can interactively explore large-scale mobility scenarios. Such tools should offer the possibility to adjust between scalability, and therefore address the question of computational time needed to compute a simulation (requiring a traffic model at macroscopic or mesoscopic level), and realism of the observed behaviors (requiring a traffic model at microscopic level). Interactive exploration makes it 
necessary to be able to zoom in a specific area and thus to switch at runtime between models. Before addressing the question of the dynamic coupling of models, these needs impose to explore the impact of coupling models of the same phenomenon at different scales.

To address this question, we present in this paper an agent-based traffic simulation in which mesoscopic and microscopic models are coupled. Through experimentation, we show that a hybridization strategy to adjust either the computational time or the variety of behavior is feasible. Simulations show the quantitative impacts of various rates of microscopic and mesoscopic models on the travel time, speed and computation times. In particular, we observe interesting results showing that in some inflow value ranges, both models behave quite similarly in terms of vehicle speed or travel time, but with a huge gain of computation time for the mesoscopic model. By dynamically switching between microscopic and mesoscopic models, it is, therefore, possible to adjust in real time the simulation to either increase its response speed or increase the variety of the observed behaviors. However, this switching will result in variations in terms of speed and time to travel.

This work is a first step towards a dynamic coupling strategy for large-scale mobility scenario. Future work will include evaluation of the strategy in the real context of the two French cities of Bordeaux and Dijon, and evaluation of the performance in context where users dynamically change the model run by the roads. At longer term, we intend to develop a middleware framework to couple models at runtime according to users inputs or specific metrics (e.g. CPU load).

\section{Acknowledgement}

This work is part of the SwITCh (Simulating the transition of transport Infrastructures Toward smart and sustainable Cities, ANR-19-CE22-0003) research project funded by the French Research Agency.

\section{References}

1. Andreas, P., Siemens, A., Ronald, K., Hartmut, K.: Coupling of concurrent macroscopic and microscopic traffic flow models using hybrid stochastic and deterministic disaggregation. In: Transportation and Traffic Theory in the 21st Century. Emerald Group Publishing Ltd. (2002)

2. Banos, A., Corson, N., Lang, C., Marilleau, N., Taillandier, P.: Multiscale modeling: Application to traffic flow. In: Agent-based Spatial Simulation with NetLogo, Vol. 2, pp. 37-62. Elsevier (2017)

3. Barceló, J.: Fundamentals of traffic simulation, vol. 145. Springer (2010)

4. Bazzan, A.L., Klügl, F.: A review on agent-based technology for traffic and transportation. The Knowledge Engineering Review 29(3), 375 (2014)

5. Bourrel, E., Henn, V.: Mixing micro and macro representations of traffic flow: a first theoretical step. In: Proc. of the 9th meeting of the Euro Working Group on Transportation. pp. 610-616 (2002)

6. Burghout, W.: Mesoscopic simulation models for short-term prediction. PREDIKT project report CTR2005 3 (2005)

7. Cervero, R., Guerra, E., Al, S.: Beyond mobility: Planning cities for people and places. Island Press (2017)

8. Chapuis, K., Taillandier, P., Gaudou, B., Drogoul, A., Daudé, E.: A multi-modal urban traffic agent-based framework to study individual response to catastrophic events. In: International Conference on Principles and Practice of Multi-Agent Systems. pp. 440-448. Springer (2018) 
9. Charypar, D., Axhausen, K.W., Nagel, K.: Event-Driven Queue-Based Traffic Flow Microsimulation. Transportation Research Record 2003(1), 35-40 (Jan 2007)

10. Derbel, O., Peter, T., Zebiri, H., Mourllion, B., Basset, M.: Modified intelligent driver model for driver safety and traffic stability improvement. IFAC Proc. Volumes 46(21), 744-749 (2013)

11. Drogoul, A., Huynh, N.Q., Truong, Q.C.: Coupling Environmental, Social and Economic Models to Understand Land-Use Change Dynamics in the Mekong Delta. Frontiers in Environmental Science 4 (2016)

12. Gazis, D.C., Herman, R., Rothery, R.W.: Nonlinear follow-the-leader models of traffic flow. Operations research 9(4), 545-567 (1961)

13. Haman, I.T., Kamla, V.C., Galland, S., Kamgang, J.C.: Towards an multilevel agent-based model for traffic simulation. Procedia Computer Science 109, 887-892 (2017)

14. Hoogendoorn, S.P., Bovy, P.H.: State-of-the-art of vehicular traffic flow modelling. Proceedings of the Institution of Mechanical Engineers, Part I: Journal of Systems and Control Engineering 215(4), 283-303 (2001)

15. Lighthill, M.J., Whitham, G.B.: On kinematic waves II. A theory of traffic flow on long crowded roads. Proceedings of the Royal Society of London. Series A. Mathematical and Physical Sciences 229(1178), 317-345 (1955)

16. Lopez, P.A., Behrisch, M., Bieker-Walz, L., Erdmann, J., Flötteröd, Y.P., Hilbrich, R., Lücken, L., Rummel, J., Wagner, P., Wießner, E.: Microscopic traffic simulation using sumo. In: 21st Int. Conference on Intelligent Transportation Systems (ITSC). pp. 2575-2582. IEEE (2018)

17. Magne, L., Rabut, S., Gabard, J.F.: Towards an hybrid macro-micro traffic flow simulation model. In: INFORMS spring 2000 meeting (2000)

18. Mohan, R., Ramadurai, G.: State-of-the art of macroscopic traffic flow modelling. International Journal of Advances in Engineering Sciences and Applied Mathematics 5(2-3), 158-176 (2013)

19. Morvan, G.: Multi-level agent-based modeling - A literature survey. arXiv:1205.0561 [cs] (Nov 2013), http://arxiv.org/abs/1205.0561, arXiv: 1205.0561

20. Pell, A., Meingast, A., Schauer, O.: Trends in real-time traffic simulation. Transportation research procedia 25, 1477-1484 (2017)

21. Popping, J.: An overview of microscopic and macroscopic traffic models. Doctoral dissertation, Faculty of Science and Engineering (2013)

22. Radu, L.D.: Disruptive technologies in smart cities: A survey on current trends and challenges. Smart Cities 3(3), 1022-1038 (2020)

23. Roads, U.S.B.o.P.: Traffic Assignment Manual for Application with a Large, High Speed Computer. U.S. Department of Commerce, Bureau of Public Roads, Office of Planning, Urban Planning Division (1964)

24. Schulze, J., Müller, B., Groeneveld, J., Grimm, V.: Agent-based modelling of social-ecological systems: achievements, challenges, and a way forward. Journal of Artificial Societies and Social Simulation 20(2) (2017)

25. Šurdonja, S., Giuffrè, T., Deluka-Tibljaš, A.: Smart mobility solutions-necessary precondition for a well-functioning smart city. Transportation research procedia 45, 604-611 (2020)

26. Taillandier, P., Gaudou, B., Grignard, A., Huynh, Q.N., Marilleau, N., Caillou, P., Philippon, D., Drogoul, A.: Building, composing and experimenting complex spatial models with the GAMA platform. GeoInformatica 23(2), 299-322 (Apr 2019)

27. Taplin, J.: Simulation models of traffic flow. The 34th Annual Conference of the Operational Research Society of New Zealand, New Zealand p. 12 (1999)

28. Tchappi Haman, I., Kamla, V.C., Galland, S., Kamgang, J.C.: Towards an Multilevel Agentbased Model for Traffic Simulation. Procedia Computer Science 109, 887-892 (2017)

29. Treiber, M., Hennecke, A., Helbing, D.: Congested traffic states in empirical observations and microscopic simulations. Physical Review E 62(2), 1805-1824 (Aug 2000)

30. Vorraa, T., Brignone, A.: Modelling traffic in detail with mesoscopic models: Opening powerful new possibilities for traffic analyses. WIT Trans. on the Built Environment 101, 659-666 (2008)

31. Wegener, M.: The future of mobility in cities: Challenges for urban modelling. Transport Policy 29, 275-282 (2013) 